\title{
Nomograms for predicting progression-free survival and overall survival after surgery and concurrent chemoradiotherapy for glioblastoma: a retrospective cohort study
}

\author{
Lin Zheng ${ }^{1,2 \#}$, Zhi-Rui Zhou ${ }^{3 \#}$, Minghan Shi ${ }^{4}$, Haiyan Chen ${ }^{1}$, Qian-Qian Yu ${ }^{1}$, Yang Yang ${ }^{1}$, Lihong Liu ${ }^{1}$, \\ Lili Zhang ${ }^{1}$, Yinglu Guo ${ }^{1}$, Xiaofeng Zhou ${ }^{1}$, Chao Li $^{1}$, Qichun Wei ${ }^{1}$ \\ ${ }^{1}$ Department of Radiation Oncology, The Second Affiliated Hospital, Zhejiang University School of Medicine, Hangzhou, China; ${ }^{2}$ Department of \\ Radiation Oncology, Taizhou Cancer Hospital, Taizhou, China; ${ }^{3}$ Radiation Oncology Center, Huashan Hospital, Shanghai Medical College, Fudan \\ University, Shanghai, China; ${ }^{4}$ Département de l'éducation aux adultes, Cégep Saint-Jean-sur-Richelieu, Brossard, QC, Canada \\ Contributions: (I) Conception and design: L Zheng, ZR Zhou, QC Wei; (II) Administrative support: QC Wei; (III) Provision of study materials or \\ patients: QC Wei, Y Yang, C Li; (IV) Collection and assembly of data: All authors; (V) Data analysis and interpretation: All authors; (VI) Manuscript \\ writing: All authors; (VII) Final approval of manuscript: All authors. \\ \#These authors contributed equally to this work. \\ Correspondence to: Qichun Wei, MD, PhD. Department of Radiation Oncology, The Second Affiliated Hospital, Zhejiang University School of \\ Medicine, Jiefang Road 88, Hangzhou 310009, China. Email: qichun_wei@zju.edu.cn.
}

Background: Glioblastoma (GBM) is the most common malignant brain tumor in adults. The prognosis of GBM patients is poor. Even with active standard treatment, the median overall survival is only 14.6 months. It is therefore critical to ascertain recurrence and search for factors that influence the prognosis of GBM. This study aimed to screen the variables related to the progression-free survival (PFS) and overall survival (OS) of GBM patients undergoing surgery and concurrent chemoradiotherapy, as well as propose a nomogram for individual risk prediction based on preoperative imaging parameters and clinicopathological variables readily available in clinical practice.

Methods: We retrospectively analyzed 114 consecutive patients with GBM who underwent surgery and concurrent chemoradiotherapy at the Second Affiliated Hospital, Zhejiang University School of Medicine from January 1st, 2015, to June 1st, 2018. Twenty-four preoperative magnetic resonance imaging (MRI) parameters were extracted manually from the Picture Archiving and Communication System (PACS). Clinicopathological factors were extracted from the electronic medical record system (EMRS). Least absolute shrinkage and selection operator (LASSO) regression and Cox regression were used for feature selection and model prediction, respectively. The models were presented using nomograms, which were applied to identify the risk of recurrence and survival according to the score. The performance of the nomograms to predict PFS and OS was tested with C-statistics, calibration plots, and Kaplan-Meier curves.

Results: The results revealed that sex, Karnofsky performance score (KPS), O6-methylglucamine-DNA methyltransferase (MGMT) protein expression, number of adjuvant chemotherapy cycles with temozolomide (TMZ), and the MRI signature effectively predicted PFS; and sex, KPS, extent of surgery, number of TMZ cycles, and MRI signature effectively predicted OS. The nomogram revealed good discriminative ability (C-statistics: 0.81 for PFS and 0.79 for OS). In the nomogram of PFS, patients with a score greater than 122 were considered to have a high risk of recurrence. In the nomogram of OS, the cutoff score were 115 and 145 , and then patients were classified as low, medium, and high risk.

Conclusions: In conclusion, our nomograms can effectively predict the risk of recurrence and survival of GBM patients and thus can be a good guide for clinical practice.

Keywords: Glioblastoma (GBM); prognosis; nomograms; magnetic resonance imaging (MRI); chemoradiotherapy

Submitted Jan 21, 2021. Accepted for publication Apr 04, 2021.

doi: 10.21037/atm-21-673

View this article at: http://dx.doi.org/10.21037/atm-21-673 


\section{Introduction}

Glioblastoma (GBM) is the most common primary malignant brain tumor in adults (1). Standard treatment includes maximal safe resection, concurrent chemoradiotherapy with temozolomide (TMZ), and six cycles of adjuvant chemotherapy $(2,3)$. GBM has a poor prognosis, with a median progression-free survival (PFS) of 9 months and overall survival (OS) of 12-15 months despite treatment with standard therapy $(4,5)$. Previous literature has reported that the risk of death was significantly reduced in selected patients who received a prolonged number of cycles of adjuvant chemotherapy (6).

Patients with GBM who receive TMZ combined with radiotherapy have a pseudoprogression rate of approximately 20-30\%. Moreover, the presence of $\mathrm{O}^{6}$-methylguanineDNA methyltransferase (MGMT) promoter methylation in tumors increases the incidence of pseudoprogression. The incidence of radiation brain necrosis is approximately $2-18 \%$ (7-9). Therefore, it is difficult to evaluate tumor recurrence based on only one magnetic resonance imaging (MRI) scan of the brain. At present, pathological diagnosis with invasive biopsy remains the gold standard to determine GBM recurrence, but it inevitably involves risk and discomfort for patients. Long-term chemotherapy is preferred for patients exhibiting sustained improvement during standard treatment $(6,10)$. However, continued long-term chemotherapy for patients who progress during treatment will bring additional financial burdens and adverse effects. An accurate, noninvasive estimation based on a riskbenefit assessment can help doctors choose an appropriate treatment strategy for patients.

In recent years, efforts have been made to predict the recurrence of GBM. Studies have evaluated the risk of recurrence based on radiomics $(11,12)$ or molecular pathology $(13,14)$. However, the parameters for radiomic prediction models need to be extracted by specific computer software. Due to variations in the instrument models and technologies of image acquisition, these results are not easy to compare among institutions, which limits their widespread use. In contrast, traditional imaging features have the advantage of being independent of image acquisition technology. Thus, the results can be easily compared among different institutions, facilitating their wide application. In addition, the prediction of tumor recurrence with molecular pathology alone is not ideal. Although several studies have evaluated molecular determinants of outcome, biomarkers for treatment stratification, and prognostic scores to predict treatment response, few have reached real clinical relevance $(3,15-17)$.

Owing to the lack of a specific and practical predictive method, it is necessary to develop a predictive model that incorporates preoperative clinicopathological data with imaging parameters. Of all the available models, nomograms can provide an individualized, evidence-based, and highly accurate risk estimation. Nomograms can be easily used and can facilitate management-related decisions. Therefore, in this study, we developed prediction models for PFS and OS with clinicopathological data and preoperative imaging parameters for GBM patients after surgery and concurrent chemoradiotherapy. We present the following article in accordance with the Tripod reporting checklist (available at http://dx.doi.org/10.21037/atm-21-673).

\section{Methods}

\section{Patient database}

From January 1, 2015, to June 1, 2018, data on consecutive GBM patients who were newly diagnosed and treatmentnaive were retrospectively collected at our department. Age, sex, Karnofsky performance score (KPS), extent of surgery, preoperative MRI parameters, Ki-67, MGMT methylation status, MGMT protein expression, and isocitrate dehydrogenase (IDH1/2) mutation status were evaluated at the beginning of radiotherapy. All patients were diagnosed pathologically by surgical resection. GBM was confirmed pathologically according to the 2016 World Health Organization (WHO) classification of central nervous system tumors, 4th edition. Histopathological sections obtained before 2016 were re-reviewed according to the latest diagnostic criteria. All patients received TMZ with concurrent radiotherapy. Clinical, pathological, and imaging data were collected from the Picture Archiving and Communication System (PACS) and electronic medical record system (EMRS) of the Second Affiliated Hospital, Zhejiang University School of Medicine. The study was approved by the institutional ethics committee of the Second Affiliated Hospital, Zhejiang University School of Medicine [No. (2020) Lunshen Yandi (032) Hao]. Informed consent was waived due to the retrospective nature of the study. The study was conducted in accordance with the Helsinki Declaration (as revised in 2013). Our research was registered in the Chinese Clinical Trial Registry (no. ChiCTR2000033266; http://www.chictr.org.cn/index. aspx). 


\section{Patient characteristics}

All 114 patients were treated with concurrent chemoradiotherapy, and the standard prescription dose of radiotherapy was $60 \mathrm{~Gy} / 30 \mathrm{fx}$. The gross tumor volume (GTV) was defined as the postoperative residual cavity and any tumor-enhancing lesions on T1-weighted MRI, excluding edema areas. Clinical tumor volume 1 (CTV1) and CTV2 were defined as the edges of the GTV extending $1 \mathrm{~cm}$ and $2 \mathrm{~cm}$, respectively. In the contouring of the CTV, edema was excluded except for the edge of enhancement. CTV1 and CTV2 were expanded by $3 \mathrm{~mm}$ to obtain the planned tumor volume 1 (PTV1) and PTV2, respectively. All treatments were carried out with intensitymodulated radiotherapy (IMRT) with prescription doses of $54 \mathrm{~Gy} / 30 \mathrm{fx}$ for PTV2 and $60 \mathrm{~Gy} / 30 \mathrm{fx}$ for PTV1. According to the standard Stupp protocol (3), concurrent TMZ chemotherapy at $75 \mathrm{mg} / \mathrm{m}^{2} / \mathrm{d}$ during radiotherapy was administered 7 days per week. Adjuvant TMZ chemotherapy was performed 4 weeks after the completion of radiotherapy. The TMZ dose was $150 \mathrm{mg} / \mathrm{m}^{2}$ in the first cycle and increased to $200 \mathrm{mg} / \mathrm{m}^{2}$ from the second cycle if chemotherapy was well tolerated. When the disease progressed or Radiation Therapy Oncology Group (RTOG) grade 3-4 toxicity occurred, the treatment regimen was changed or the dose of chemotherapy was reduced. It took 9 months for patients to complete the standard six cycles of adjuvant chemotherapy, so we predicted the recurrence probability of patients at 9 months, which was the conclusion of the standard therapy process, to guide the decision regarding continued long-term chemotherapy. The endpoints of this study were PFS and OS, which were defined as the time intervals from the patient's surgical treatment to recurrence and death or the last follow-up, respectively.

\section{MRI}

Preoperative and postoperative MRIs from PACS were evaluated. The following preoperative MRI-relevant sequences were assessed: T1-weighted, T1-weighted gadolinium contrast-enhanced, T2-weighted, T2-weighted fluid-attenuated inversion recovery (FLAIR), and diffusionweighted imaging (DWI). The imaging parameters referred to the semantic visually accessible Repository of Molecular Brain Neoplasia Data (REMBRANDT) images (VASARI) imaging feature guide v.1.1. (https:// wiki.nci.nih.gov/display/CIP/VASARI) (18). Twenty-four preoperative imaging parameters were extracted, including tumor location, side of tumor epicenter (bilateral or onesided), eloquent brain, enhancement quality, proportions of contrast-enhancing tumor (CET)/non-contrast-enhancing tumor (nCET)/edema/necrosis, cysts, multifocality or multicentricity, T1/FLAIR ratio, thickness of enhancing margin, definition of enhancing margin, definition of non-enhancing margin, CET/nCET/edema crossing the midline, hemorrhage, diffusion, pial invasion, ependymal invasion, cortical involvement, deep white-matter (wm) invasion, and satellites. All preoperative imaging data were independently assessed by two experienced neurooncology radiologists. In circumstances where a unanimous agreement could not be reached, a third radiologist with 20 years of professional experience in neuroradiology was consulted. After discussion, the final standard radiology report of each patient was generated. Except for the diagnostic information of GBM, the radiologists were blind to any clinical and molecular pathologic information of the patients.

\section{Tumor recurrence}

Tumor recurrence was determined by at least one of the following four conditions: (I) continuous follow-up enhanced MRI scans of the brain were performed multiple times, and these scans indicated tumor recurrence; (II) MR perfusion-weighted imaging (PWI), magnetic resonance spectroscopy (MRS), DWI and other examination methods, clinical symptoms, and the diagnostic conclusion of image recurrence were assessed comprehensively; (III) tumor recurrence was confirmed by reoperation and pathology; (IV) tumor recurrence of some difficult cases was decided after multidisciplinary team (MDT) discussion.

\section{Statistical analysis}

Some continuous variables were transformed into rank variables for analysis. For example, the percentage of the enhanced portion in the tumor volume was scored as follows: $1=0 \% ; 2=\leq 5 \% ; 3=6-33 \% ; 4=34-67 \% ; 5=$ $68-95 \% ; 6=\geq 95 \% ; 7=100 \%$. We transformed continuous variables into rank variables or two-category variables according to the interquartile spacing/median. Some of the data were divided into two categories for analysis. Categorical variables are reported as integers and proportions, and continuous variables are reported as medians with interquartile ranges (IQRs). The PFS and OS 

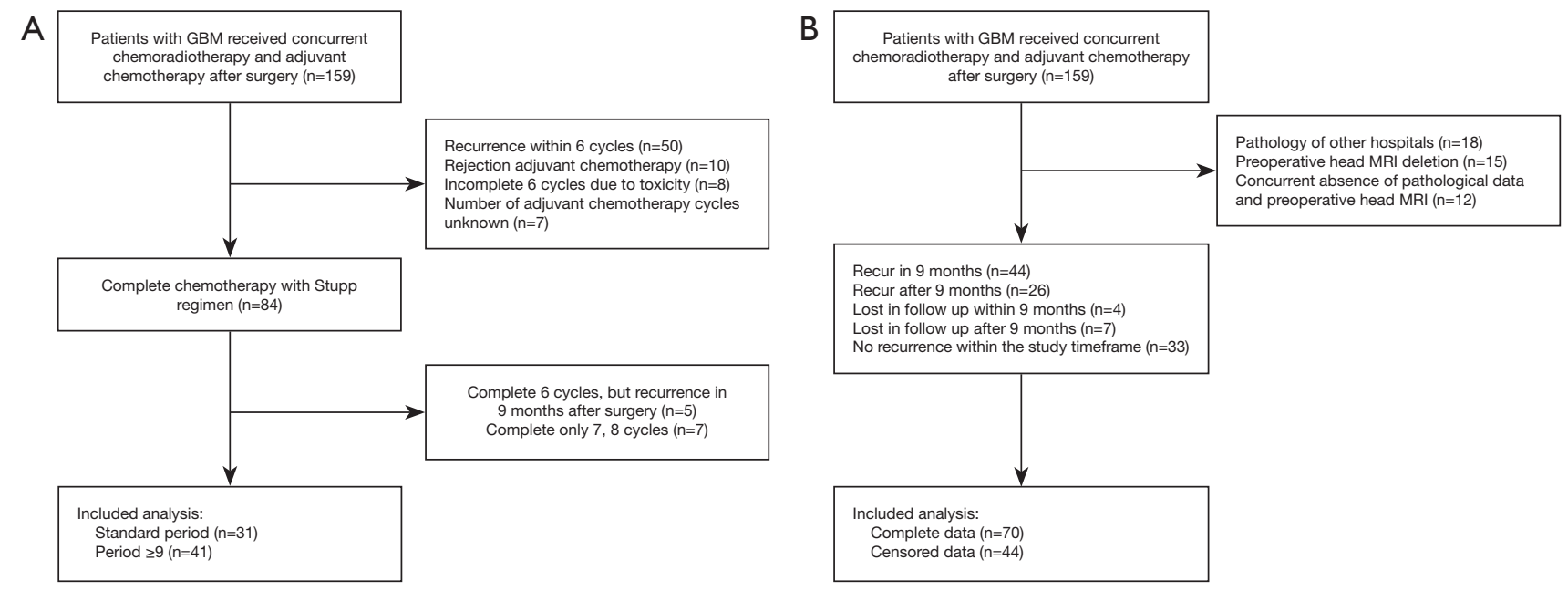

Figure 1 Flowchart of the study. (A) Flowchart of the Stupp protocol selection process. (B) Flowchart of the study selection process. GBM, glioblastoma; MRI, magnetic resonance imaging.

for the study population were assessed using the KaplanMeier method with the log-rank test. Clinicopathological variables associated with recurrence and survival risk were assessed based on their clinical importance as identified in previously published articles (19-22), $\mathrm{P}$ values, and sample size. A regularization method was conducted to solve the problem of overfitting caused by multicollinearity. Variables with no interaction were included in the multivariate analysis. A least absolute shrinkage and selection operator (LASSO) regression cross-validation was used to select the MRI imaging parameters and to screen out meaningful variables. On consideration of the $\mathrm{P}$ value and clinical importance of variables, the Cox regression equation was finally calculated. A multivariate Cox proportional hazards regression model was used to evaluate independent prognostic factors affecting PFS and OS and to calculate the hazard ratio (HR) values and 95\% confidence intervals (CIs). Selected variables were incorporated in the nomograms to predict the 9-month, 2-year, and 3-year PFS rates and the 1-, 2-, and 3-year OS rates after resection and concurrent chemoradiotherapy for GBM using statistical software (rms in R, version 3.5.2; http://www.r-project.org/). $\mathrm{C}$-statistics were used to measure the discrimination ability of the nomograms. Calibration curves were drawn by bootstrap validation with 1,000 resamplings to determine the degree of coincidence between the actual probability and the predicted probability. Survival curves were calculated for the clinical efficacy analysis.

All tests were two-sided, and $\mathrm{P}<0.05$ was considered statistically significant. All analyses were performed using $\mathrm{R}$, version 3.5.2. The deadline for data analysis was May 1 , 2019.

\section{Results}

\section{Clinical features, PFS, OS, and adverse drug reactions}

Among the 159 patients, 50 did not complete the six cycles of adjuvant chemotherapy due to disease progression, 10 refused to continue chemotherapy, 8 were suspended due to RTOG grade 3-4 toxicity, and 7 received an unknown number of adjuvant chemotherapy cycles. Eighty-four patients completed the Stupp protocol, 41 of whom received $\geq 9$ cycles of adjuvant chemotherapy (Figure 1A).

Patients who did not undergo preoperative head MRI and whose clinicopathological data were lacking because surgery was performed at other hospitals were excluded, and 114 patients were finally included in the analysis (Figure 1B). According to the surgical records and enhanced MRI within 72 hours after surgery, 55 patients were judged as having total tumor resection, 55 patients were judged as having subtotal tumor resection, and 4 patients were biopsied only. There were 68 males and 46 females. The median age was 58 years (range, $21-80$ years). The patients had a median KPS of 90 (range, 50-90) at the beginning of radiotherapy. MGMT protein expression test results were obtained in all 114 patients, and MGMT promoter region methylation was obtained in 34 specimens. Both PCR and immunohistochemistry obtained MGMT results 
Table 1 Clinical characteristics of patients with GBM ( $n=114)$

\begin{tabular}{|c|c|}
\hline Characteristics & N (\%) \\
\hline \multicolumn{2}{|l|}{ Sex } \\
\hline Male & $68(59.65)$ \\
\hline Female & $46(40.35)$ \\
\hline \multicolumn{2}{|l|}{ Age (years) } \\
\hline Median (range) & 58 [21-80] \\
\hline$<50$ & $33(28.95)$ \\
\hline$\geq 50$ & $81(71.05)$ \\
\hline \multicolumn{2}{|c|}{ Karnofsky performance score } \\
\hline Median [range] & 90 [50-90] \\
\hline$<70$ & $5(13.16)$ \\
\hline$\geq 70$ & $99(86.84)$ \\
\hline \multicolumn{2}{|l|}{ Extent of surgery } \\
\hline Complete resection & $55(48.25)$ \\
\hline Partial resection & $5(48.25)$ \\
\hline Biopsy & $4(3.50)$ \\
\hline \multicolumn{2}{|l|}{ Pathological diagnosis } \\
\hline Glioblastoma & 114 \\
\hline \multicolumn{2}{|l|}{ Ki-67 } \\
\hline$\leq 25 \%$ & $37(32.46)$ \\
\hline $26-50 \%$ & $65(57.02)$ \\
\hline $51-75 \%$ & $8(7.02)$ \\
\hline$>75 \%$ & $4(3.50)$ \\
\hline \multicolumn{2}{|c|}{ MGMT protein expression } \\
\hline Negative & $88(77.19)$ \\
\hline Positive & $26(22.81)$ \\
\hline \multicolumn{2}{|l|}{ IDH1/2 status } \\
\hline Wild-type & $95(83.30)$ \\
\hline Mutant & $9(16.70)$ \\
\hline
\end{tabular}

GBM, glioblastoma; MGMT, $\mathrm{O}^{6}$-methylguanine-DNA methyltransferase; IDH, isocitrate dehydrogenase.

in 34 patients. Of the 29 MGMT promoter methylation samples, 25 were negative for MGMT protein, three were suspiciously positive, and one was positive. Of the five samples with an unmethylated MGMT promoter region, one was negative, one was suspiciously positive, and three were positive for protein expression. The IDH mutation status of 19 patients was positive (Table 1).

A specific recurrence time was determined in 70 of 114 patients. Among them, 14 cases were confirmed by reoperation, and 15 cases were difficult to diagnose and considered to have tumor recurrence after MDT discussion. The median follow-up period was 17.63 months (range, 3.47-47.33 months), of which $61.40 \%(70 / 114)$ of patients experienced recurrence and $59.65 \%(68 / 114)$ of patients died. The median PFS and OS were 14.4 months (95\% CI, 11.2-19.0 months) and 21.1 months (95\% CI, 17.2-26.6 months), respectively. The 9-month, 2-year, and 3-year PFS rates were $63.2 \%$ (95\% CI, 54.9-72.9\%), 26.0\% (95\% CI, 17.4-38.8\%) and $20.6 \%$ (95\% CI, 12.0-33.4\%), respectively. The 1-, 2-, and 3 -year OS rates were 79.8\% (95\% CI, 72.8-87.5\%), 38.7\% (95\% CI, 30.1-49.9\%) and 30.4\% (95\% CI, $21.5-42.8 \%$ ), respectively (Figure $2 A$ ).

Among the 84 patients who completed the Stupp protocol, 31 patients completed the standard six cycles of chemotherapy and did not experience recurrence. Fortyone patients completed adjuvant chemotherapy with more than or equal to 9 cycles (Figure $1 A$ ). Patients with $\geq 9$ cycles of chemotherapy had a longer OS (43.0 months, $95 \%$ CI, 29-NA months) than patients with six standard cycles (26.0 months, 95\% CI, 18-NA months), with $\mathrm{P}=0.041$ (Figure 2B). There were no significant differences in the comparison of adverse reactions between the long period and the standard period groups (Table 2).

\section{Independent prognostic factors}

A total of 24 imaging parameters were included in the model, and potential predictive variables were obtained by using a LASSO regression cross-validation dimension reduction. The variables obtained were features with nonzero coefficients in the LASSO regression model (Figures 2C,D). By linearly combining the features with nonzero coefficients, the PFS and OS MRI signatures could be constructed, and the MRI imaging signature scores could be computed as follows:

PFS MRI signature score $=$ enhancement quality $\times$ $(0.19413430)+$ multifocality or multicentricity $\times(0.04539098)$ + ependymal invasion $\times(0.15332560)+$ satellites $\times$ (0.76285505).

OS MRI signature score $=$ tumor location $\times 0.021706308$ + enhancement quality $\times 0.263196546+$ proportion of noncontrast-enhancing tumor $\times 0.007577829+$ multifocality or multicentricity $\times 0.041631945+$ ependymal invasion $\times$ $0.230683569+$ cortical involvement $\times(-0.246257526)+$ 

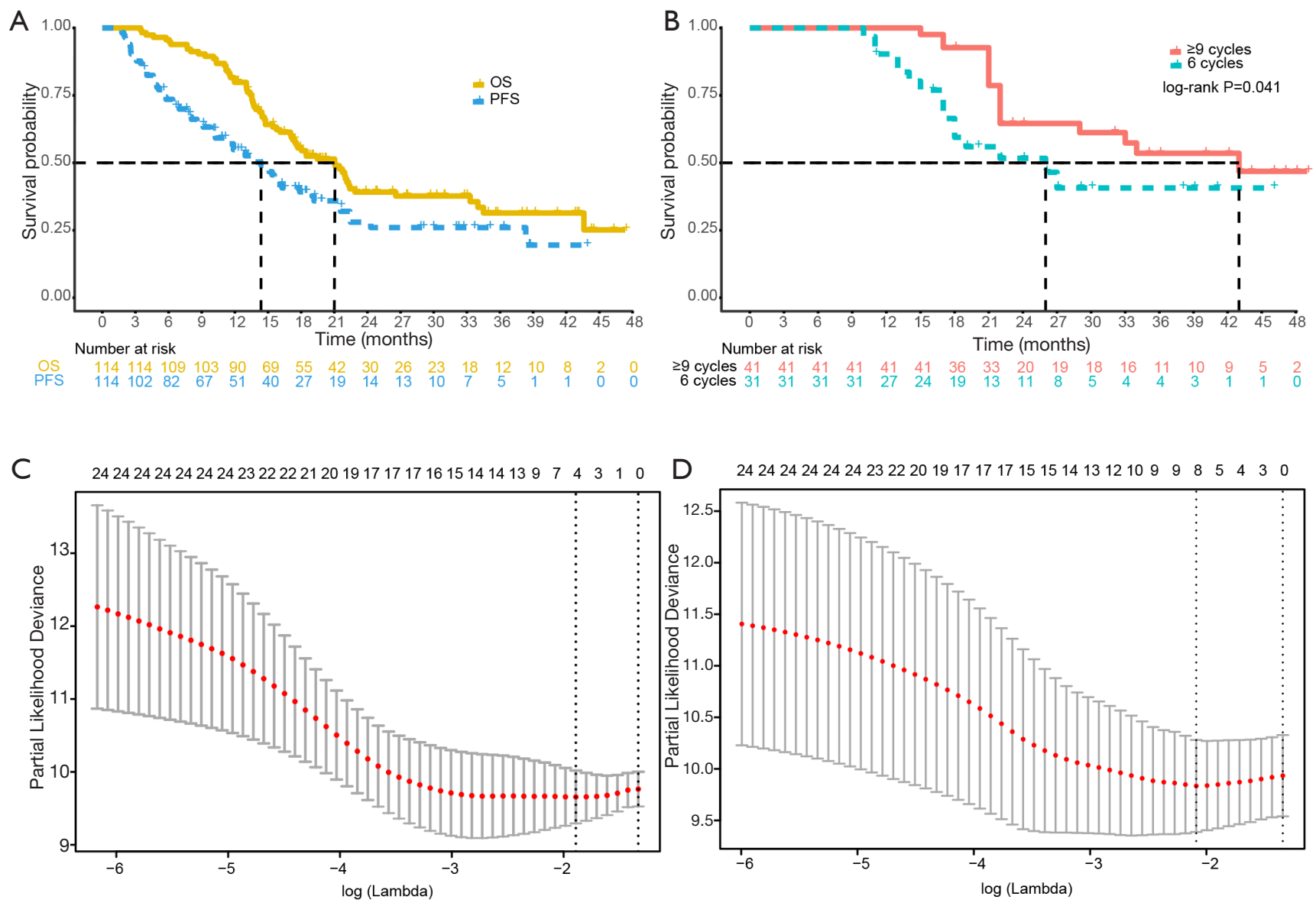

E

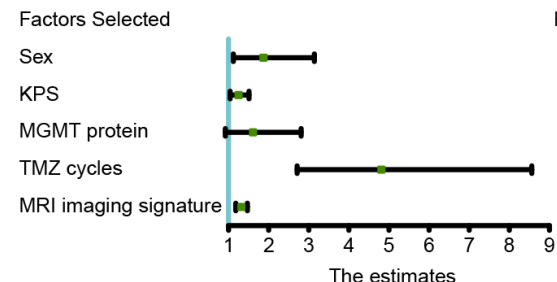

F

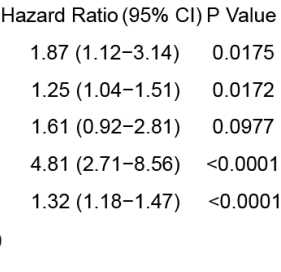

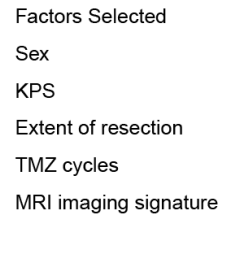

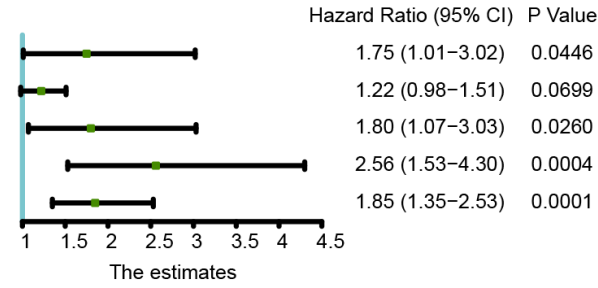

Figure 2 Independent prognostic factors. (A) OS and PFS of 114 GBM patients. (B) OS of GBM patients with six cycles of chemotherapy (blue line) and more than or equal to 9 cycles of chemotherapy (red line); P=0.041. (C) LASSO regression cross-validation for predicting PFS. (D) LASSO regression cross-validation for predicting OS. (E) The forest plot of variables with the strongest correlation with the risk of PFS. (F) The forest plot of variables with the strongest correlation with the risk of OS. PFS, progression-free survival; OS, overall survival; KPS, Karnofsky performance score; MGMT, O6-methylguanine-DNA methyltransferase; TMZ, temozolomide; MRI, magnetic resonance imaging; GBM, glioblastoma; LASSO, least absolute shrinkage and selection operator.

contrast-enhancing tumor crosses midline $\times(0.119200807)$ + satellites $\times(0.461748899)$.

The clinicopathological variables were analyzed by univariate analysis (Table 3). Then, multivariate Cox proportional hazards regression analysis was performed to screen out the five variables demonstrating the strongest correlation with the risk of recurrence. The five independent prognostic factors of PFS were sex, KPS, MGMT protein expression, TMZ cycles, and MRI signature (Figure 2E). Multivariate Cox proportional hazards regression was used to obtain a $\mathrm{C}$ index of 0.81 (95\% CI, 0.77-0.86). Similarly, the Cox proportional hazards 
Table 2 Comparison of adverse reactions in patients with GBM in the long period (more than or equal to 9 cycles of chemotherapy) and standard period (6 cycles of chemotherapy) groups $(\mathrm{n}=84)$

\begin{tabular}{|c|c|c|c|c|}
\hline Adverse reactions & Long period $(n=48)(\%)$ & Standard period $(n=36)(\%)$ & $\chi^{2}$ & $P$ value \\
\hline \multicolumn{5}{|l|}{ Leukopenia } \\
\hline $\mathrm{I} / \mathrm{II}$ & $12(25.00)$ & $9(25.00)$ & 0.00 & 1.00 \\
\hline III/IV & $0(0.00)$ & $1(2.78)$ & & 0.43 \\
\hline \multicolumn{5}{|l|}{ Thrombocytopenia } \\
\hline III/IV & $1(2.08)$ & $0(0.00)$ & & 1.00 \\
\hline \multicolumn{5}{|l|}{ Hemoglobin decline } \\
\hline $\mathrm{I} / \mathrm{II}$ & $11(22.92)$ & $7(19.44)$ & 0.15 & 0.70 \\
\hline III/IV & $1(2.08)$ & $1(2.78)$ & & 1.00 \\
\hline III/IV & $5(10.42)$ & $1(2.78)$ & 0.84 & 0.36 \\
\hline
\end{tabular}

GBM, glioblastoma.

Table 3 Univariate analysis of predictors of progression-free survival and overall survival

\begin{tabular}{|c|c|c|c|c|}
\hline Variable & \multicolumn{2}{|c|}{ PFS Cox regression univariable } & \multicolumn{2}{|c|}{ OS Cox regression univariable } \\
\hline Sex & $1.53(0.93-2.50)$ & 0.0951 & $1.64(0.99-2.72)$ & 0.0536 \\
\hline Age & 1.39 (1.01-1.90) & 0.0408 & $1.26(0.92-1.73)$ & 0.1456 \\
\hline KPS & $1.29(1.07-1.55)$ & 0.0086 & $1.29(1.06-1.56)$ & 0.0099 \\
\hline Ki-67 & $1.04(0.78-1.38)$ & 0.7859 & $1.14(0.86-1.52)$ & 0.3487 \\
\hline MGMT protein & $1.50(0.87-2.57)$ & 0.1422 & $1.15(0.66-2.02)$ & 0.6233 \\
\hline IDH & $1.49(0.76-2.91)$ & 0.2439 & $1.09(0.58-2.03)$ & 0.7967 \\
\hline TMZ cycles & $5.21(3.02-8.99)$ & $<0.0001$ & $3.20(1.95-5.24)$ & $<0.0001$ \\
\hline
\end{tabular}

PFS, progression-free survival; OS, overall survival; HR, hazard ratio; KPS, Karnofsky performance score; MGMT, ${ }^{6}$-methylguanine-DNA methyltransferase; IDH, isocitrate dehydrogenase; TMZ, temozolomide.

regression model identified five variables with the strongest correlation with OS, including sex, KPS, extent of resection, TMZ cycles, and MRI imaging signature (Figure $2 F$ ), and the $\mathrm{C}$ index was 0.79 (95\% CI, 0.75-0.84).

\section{Development and validation of an individualized prediction model}

These independently correlated risk factors were used to construct nomograms for tumor recurrence risk prediction and mortality risk prediction (Figures $3 A, B$ ). The higher the total score according to the sum of the specified points of each variable in the nomogram, the worse the prognosis. For example, patient No. 37 was a female with a KPS of 80, MGMT protein expression positive, six cycles of adjuvant chemotherapy, and a preoperative MRI signature score of 1.7 points. Her total score was 107 , and the tumor recurrence probability at 9 months was $70 \%$. Similarly, for 

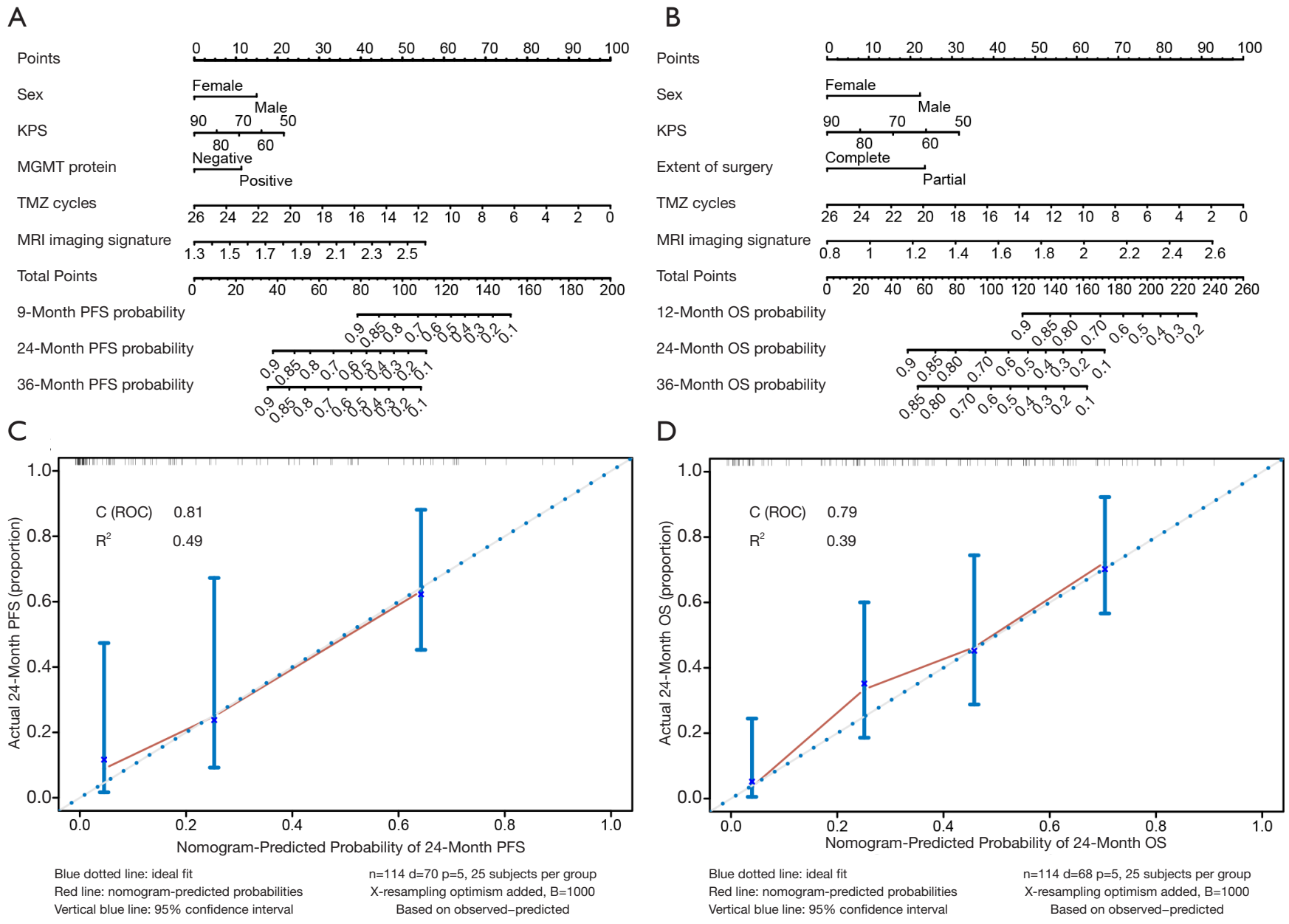

Figure 3 Individualized prediction model. (A) Nomogram to predict PFS at 9, 24, and 36 months. (B) Nomogram to predict OS at 12, 24, and 36 months. (C,D) The nomogram-predicted probabilities of PFS and OS within 24 months are plotted on the $\mathrm{x}$-axis. The actual probabilities of PFS and OS are plotted on the y-axis. KPS, Karnofsky performance score; MGMT, O6-methylguanine-DNA methyltransferase; TMZ, temozolomide; MRI, magnetic resonance imaging; PFS, progression-free survival; OS, overall survival; GBM, glioblastoma; C index, concordance index; ROC, receiver operating characteristic.

patient No. 41, his basic information was as follows: male, KPS 90, resection completion, nine cycles of adjuvant chemotherapy, preoperative MRI imaging signature score of 1.34 points. He received a total score of 114 points, and the probability of survival was predicted to be $>90 \%$ at 1 year, $60 \%$ at 2 years, and $51 \%$ at 3 years. In actuality, he died 21.47 months after diagnosis. The internal validation of the model was carried out by the bootstrap sample validation method. The nomogram demonstrated good accuracy in estimating the risk of PFS and OS, with unadjusted $\mathrm{C}$ indexes of 0.81 (95\% CI, 0.77-0.86) and 0.79 (95\% CI, $0.75-0.84)$, respectively, and bootstrap-corrected $\mathrm{C}$ indexes of 0.80 and 0.78 . In addition, the calibration curves showed that the risk of recurrence and survival predicted by the nomograms were in good agreement with the actual data (Figures 3C,D).

\section{Clinical efficacy analysis}

To evaluate the ability of the model to predict PFS, the nomogram score of each patient was calculated. The optimal cutoff value of the total nomogram score was determined to be 122 using X-tile (23). Based on this threshold, 36 patients (31.58\%) with a total score of more than 122 were classified as the high risk of recurrence group, and the remaining patients were classified as the 

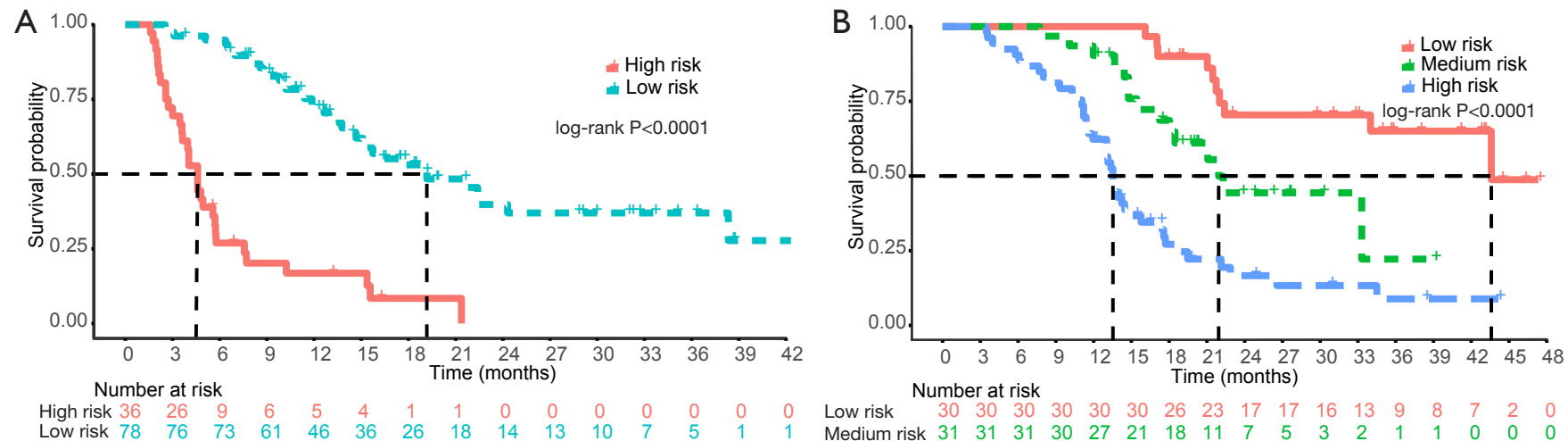
$\begin{array}{llllllllllllll}\text { High risk } 36 & 26 & 9 & 6 & 5 & 4 & 1 & 1 & 0 & 0 & 0 & 0 & 0 & 0 \\ \text { Low risk } 78 & 76 & 73 & 61 & 46 & 36 & 26 & 18 & 14 & 13 & 10 & 7 & 5 & 1\end{array}$

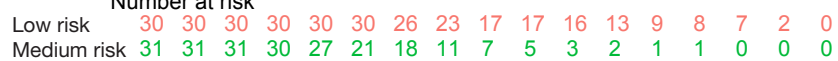
$\begin{array}{llllllllllllllllll}\text { High risk } & 53 & 53 & 48 & 43 & 33 & 18 & 11 & 8 & 6 & 4 & 4 & 3 & 2 & 1 & 1 & 0 & 0\end{array}$

Figure 4 Survival risk stratification for patients with GBM. (A) Progression-free survival risk stratification for patients with GBM. (B) Overall survival risk stratification for patients with GBM. GBM, glioblastoma.

low risk of recurrence group. The predicted probabilities of PFS were plotted as a Kaplan-Meier curve (Figure 4A). Similarly, the predicted probabilities of OS were plotted as a Kaplan-Meier curve, and the total score calculated by the nomogram was divided into three groups by using $\mathrm{X}$-tile, with boundary values of 115 and 145 points. Thirty patients $(26.32 \%)$ had scores of less than 115 points, 31 (27.19\%) had scores between 115 and 145 points, and 53 (46.49\%) had scores of more than 145 points; these patients were classified into the low, medium, and high risk of death groups, respectively. The median 2-year OS values predicted by the nomogram were $69.66 \%, 40.97 \%$, and $15.63 \%$ for the low, medium, and high risk of death groups, respectively $(\mathrm{P}<0.0001)$ (Figure $4 B)$.

\section{Discussion}

In this study, in consideration of convenience and practicality, we extracted clinically accessible features and constructed two nomograms. By combining imaging features with clinicopathological features, comprehensive models were developed to improve the predictive performance. The nomograms can accurately and intuitively predict the recurrence probability and survival probability of patients. The calibration curve demonstrated that our nomograms have good predictive performance. Additionally, it was confirmed that OS significantly improved if patients received more than or equal to 9 cycles of adjuvant chemotherapy compared with standard cycles of adjuvant chemotherapy. Furthermore, there were no statistically significant differences in adverse reactions between longterm cycle chemotherapy and standard cycle chemotherapy.
$\mathrm{X}$-tile was used to obtain the cutoff value of the score, which was used to divide the patients into high and low risk of recurrence group. The patients were also divided into low, medium, and high risk of death groups according to the nomogram score. Abnormal lesions in follow-up MRI were more likely to reflect pseudoprogression in the low risk of recurrence group and true progression in the high risk of recurrence group. Therefore, we recommend continuing chemotherapy in the low risk of recurrence group when abnormal lesions are detected on the first MRI scan and switching to other treatments in the high risk of recurrence group.

Previous studies have tried to predict PFS and OS with radiomics, but it is not widely used because the parameters of these prediction models need to be extracted by specific computer software and cannot be realized by routine examination, which limits its widespread use and makes it impossible for clinical application. Also, radiology features can be easily affected by image acquisition details, such as scanner types, separate protocols, and sequence specifications, and radiomic results are difficult to compare because of the lack of standardized methods to guide the quantitative analysis of images (24). In contrast, semantic image systems depend less on image acquisition instruments and technical specifications. As a result, our data can be more easily compared in different institutions, thereby facilitating their widespread use. Moreover, the lack of specificity in the biological sense and fuzziness in interpretation may be one of the limitations of radiology. Radiological characteristics are "agnostic" insofar as they are difficult to explain or describe intuitively, although radiological characteristics are known to reflect intragenic 
heterogeneity and invasiveness $(25,26)$. Our study shows that traditional MRI-based radiological features combined with clinicopathological factors can predict recurrence and prognosis well.

As the current gold standard, biopsy is required to identify lesions as pseudoprogression, radioactive brain necrosis, or true progression, but it certainly results in more stress and pain for the patient. Although these states can also be determined by noninvasive methods, MRS, DWI, PWI and other examinations as well as an MDT discussion are required, which demand human and material resources and increase the economic burden. This study is primarily designed for clinical work, and our findings can increase convenience and decrease costs. We accurately predicted the probability of recurrence at 9 months, 2 years, and 3 years in a noninvasive manner, which can guide followup treatment and avoid wasting resources and adverse reactions to chemotherapy caused by false negatives or losing long-term chemotherapy opportunities caused by false positives. With the predicted PFS and OS of patients, healthcare providers can inform patients about their prognosis, explain their conditions, and make personalized decisions on treatment and monitoring. As a quantitative tool for assessing risk and benefit, the clinical prediction model can provide more objective and accurate information for the decision-making of doctors, patients, and health administrators (27).

The PFS and OS of the patients enrolled in this study were better than those reported in the Stupp study (3), likely due to the following reasons. (I) These patients all completed concurrent chemoradiotherapy, and the patients with lower KPS were not included in this study. (II) Only $47 \%$ of the patients in the Stupp study (3) completed 6 cycles of adjuvant chemotherapy. In our group, 58.8\% $(67 / 114)$ of patients completed 6 cycles of adjuvant chemotherapy, and $28.9 \%$ (33/114) of patients received more than or equal to 9 cycles of chemotherapy. (III) The proportion of patients with the IDH mutation, which is associated with good prognosis, was as high as $16.7 \%$.

Although our study demonstrated that our models can accurately predict prognosis, they still have some limitations. First, this study was a retrospective study with a relatively small sample size. A prospective study is needed to confirm the reliability of our nomograms. Second, it was based on single-center data, and although consistent settings can improve the effectiveness of the technology and control confounding factors, this approach inevitably narrows the scope of application. In the future, data from multiple centers should be used to build prediction models with better generality. Third, although our nomograms were verified internally through resampling verification, further research is needed to verify the reliability of the proposed nomograms with external verification.

In conclusion, we constructed PFS and OS prediction models for GBM with parameters that are easily accessible in clinical settings. Our nomograms can intuitively predict the recurrence and survival probabilities of patients with excellent predictive performance. They are able to stratify patients into recurrence risk groups and prognostic groups to guide follow-up treatment and they performed well on internal validation. Therefore, the proposed predictive models have the potential to improve the management of GBM patients. Further studies are needed to externally validate the proposed nomograms to determine their value in predicting PFS and OS after resection and concurrent chemoradiotherapy in GBM patients.

\section{Acknowledgments}

Funding: This work was supported by the Chinese Society of Neuro-oncology, CACA grant (CSNO-2014-MSD05), the Key Discipline Foundation for Radiation Oncology of Wenling City, the Taizhou Science and Technology Program (No. 20ywb138) and the National Natural Science Foundation of China (grant 82003231 to Dr. ZRZ).

\section{Footnote}

Reporting Checklist: The authors have completed the TRIPOD reporting checklist. Available at http://dx.doi. org/10.21037/atm-21-673

Data Sharing Statement: Available at http://dx.doi. org/10.21037/atm-21-673

Conflicts of Interest: All authors have completed the ICMJE uniform disclosure form (available at http://dx.doi. org/10.21037/atm-21-673). The authors have no conflicts of interest to declare.

Ethical Statement: The authors are accountable for all aspects of the work in ensuring that questions related to the accuracy or integrity of any part of the work are appropriately investigated and resolved. The study was approved by the institutional ethics committee of the Second Affiliated Hospital, Zhejiang University School of 
Medicine (No. (2020) Lunshen Yandi (032) Hao). Informed consent was waived due to the retrospective nature of the study. The study was conducted in accordance with the Helsinki Declaration (as revised in 2013).

Open Access Statement: This is an Open Access article distributed in accordance with the Creative Commons Attribution-NonCommercial-NoDerivs 4.0 International License (CC BY-NC-ND 4.0), which permits the noncommercial replication and distribution of the article with the strict proviso that no changes or edits are made and the original work is properly cited (including links to both the formal publication through the relevant DOI and the license). See: https://creativecommons.org/licenses/by-nc-nd/4.0/.

\section{References}

1. Ostrom QT, Gittleman H, Fulop J, et al. CBTRUS Statistical Report: Primary Brain and Central Nervous System Tumors Diagnosed in the United States in 20082012. Neuro-oncology 2015;17:iv1-62.

2. Stupp R, Mason WP, van den Bent MJ, et al. Radiotherapy plus concomitant and adjuvant temozolomide for glioblastoma. N Engl J Med 2005;352:987-96.

3. Stupp R, Hegi ME, Mason WP, et al. Effects of radiotherapy with concomitant and adjuvant temozolomide versus radiotherapy alone on survival in glioblastoma in a randomised phase III study: 5-year analysis of the EORTC-NCIC trial. Lancet Oncol 2009;10:459-66.

4. Oh J, Henry RG, Pirzkall A, et al. Survival analysis in patients with glioblastoma multiforme: predictive value of choline-to- $\mathrm{N}$-acetylaspartate index, apparent diffusion coefficient, and relative cerebral blood volume. J Magn Reson Imaging 2004;19:546-54.

5. Marijnen CA, van den Berg SM, van Duinen SG, et al. Radiotherapy is effective in patients with glioblastoma multiforme with a limited prognosis and in patients above 70 years of age: a retrospective single institution analysis. Radiother Oncol 2005;75:210-6.

6. Darlix A, Baumann C, Lorgis V, et al. Prolonged administration of adjuvant temozolomide improves survival in adult patients with glioblastoma. Anticancer Res 2013;33:3467-74.

7. Smith EA, Carlos RC, Junck LR, et al. Developing a clinical decision model: MR spectroscopy to differentiate between recurrent tumor and radiation change in patients with new contrast-enhancing lesions. AJR Am J Roentgenol 2009;192:W45-52.
8. Kong DS, Kim ST, Kim EH, et al. Diagnostic dilemma of pseudoprogression in the treatment of newly diagnosed glioblastomas: the role of assessing relative cerebral blood flow volume and oxygen-6-methylguanine-DNA methyltransferase promoter methylation status. Am J Neuroradiol 2011;32:382-7.

9. Lee WJ, Choi SH, Park CK, et al. Diffusion-weighted MR imaging for the differentiation of true progression from pseudoprogression following concomitant radiotherapy with temozolomide in patients with newly diagnosed highgrade gliomas. Acad Radiol 2012;19:1353-61.

10. Hau P, Koch D, Hundsberger T, et al. Safety and feasibility of long-term temozolomide treatment in patients with high-grade glioma. Neurology 2007;68:688-90.

11. Kickingereder $P$, Burth $S$, Wick A, et al. Radiomic Profiling of Glioblastoma: Identifying an Imaging Predictor of Patient Survival with Improved Performance over Established Clinical and Radiologic Risk Models. Radiology 2016;280:880-9.

12. McGarry SD, Hurrell SL, Kaczmarowski AL, et al. Magnetic Resonance Imaging-Based Radiomic Profiles Predict Patient Prognosis in Newly Diagnosed Glioblastoma Before Therapy. Tomography 2016;2:223-8.

13. Dubbink HJ, Taal W, Marion R, et al. IDH1 mutations in low-grade astrocytomas predict survival but not response to temozolomide. Neurology 2009;73:1792-5.

14. Carrillo JA, Lai A, Nghiemphu PL, et al. Relationship between tumor enhancement, edema, IDH1 mutational status, MGMT promoter methylation, and survival in glioblastoma. Am J Neuroradiol 2012;33:1349-55.

15. Combs SE, Edler L, Rausch R, et al. Generation and validation of a prognostic score to predict outcome after re-irradiation of recurrent glioma. Acta Oncol 2013;52:147-52.

16. Muth C, Rubner Y, Semrau S, et al. Primary glioblastoma multiforme tumors and recurrence: Comparative analysis of the danger signals HMGB1, HSP70, and calreticulin. Strahlenther Onkol 2016;192:146-55.

17. Kessel KA, Hesse J, Straube C, et al. Validation of an established prognostic score after re-irradiation of recurrent glioma. Acta Oncol 2017;56:422-6.

18. Wiki for the VASARI feature set. The National Cancer Institute Web site. 2019. Available online: https://wiki.cancerimagingarchive.net/display/Public/ VASARI+Research+Project

19. Pope WB, Sayre J, Perlina A, et al. MR imaging correlates of survival in patients with high-grade gliomas. Am J Neuroradiol 2005;26:2466-74. 


\section{Page 12 of 12}

20. Park JK, Hodges T, Arko L, et al. Scale to predict survival after surgery for recurrent glioblastoma multiforme. J Clin Oncol 2010;28:3838-43.

21. Lacroix M, Abi-Said D, Fourney DR, et al. A multivariate analysis of 416 patients with glioblastoma multiforme: prognosis, extent of resection, and survival. J Neurosurg 2001;95:190-8.

22. Hammoud MA, Sawaya R, Shi W, et al. Prognostic significance of preoperative MRI scans in glioblastoma multiforme. J Neurooncol 1996;27:65-73.

23. Camp RL, Dolled-Filhart M, Rimm DL. X-tile: a new bio-informatics tool for biomarker assessment and outcome-based cut-point optimization. Clin Cancer Res 2004;10:7252-9.

Cite this article as: Zheng L, Zhou ZR, Shi M, Chen H, Yu QQ, Yang Y, Liu L, Zhang L, Guo Y, Zhou X, Li C, Wei Q. Nomograms for predicting progression-free survival and overall survival after surgery and concurrent chemoradiotherapy for glioblastoma: a retrospective cohort study. Ann Transl Med 2021;9(7):571. doi: 10.21037/atm-21-673

\section{Zheng et al. Nomogram of predicting glioblastoma prognosis}

24. Peeken JC, Hesse J, Haller B, et al. Semantic imaging features predict disease progression and survival in glioblastoma multiforme patients. Strahlenther Onkol 2018;194:580-90.

25. Lambin P, Rios-Velazquez E, Leijenaar R, et al. Radiomics: extracting more information from medical images using advanced feature analysis. Eur J Cancer 2012;48:441-6.

26. Gillies RJ, Kinahan PE, Hricak H. Radiomics: Images Are More than Pictures, They Are Data. Radiology 2016;278:563-77.

27. Zhou ZR, Wang WW, Li Y, et al. In-depth mining of clinical data: the construction of clinical prediction model with R. Ann Transl Med 2019;7:796. 